\title{
USO DE SOFTWARE PARA ANÁLISE DE IMAGEM EM AVALIAÇÃO DA COBERTURA DE PULVERIZAÇÃO ${ }^{(1)}$
}

\author{
MÁRCIO CARVALHO FIRVEDA ${ }^{(2)}$; ALÉCIO CANTALOGO-JUNIOR ${ }^{(2)}$; HAMILTON HUMBERTO \\ RAMOS $^{(3)}$; ANTONIO CARLOS LOUREIRO LINO(3); \\ ILA MARIA CORRÊA ${ }^{(3)}$
}

\begin{abstract}
RESUMO
O presente trabalho teve como objetivo avaliar a menor concentração do traçador fluorescente Poliglow 830 YLS, necessária para um suficiente contraste com a superfície vegetal, bem como desenvolver uma rotina para determinar a cobertura de pulverização no software para análise de imagens IDRISI. Para avaliação, misturaram-se, previamente, três doses de traçador fluorescente Poliglow 830 YLS (5, 12,5 e 25 g) a 10 g de uma formulação de mancozeb, diluídas em 5 L de água $(0,001,0,0025$ e $0,005 \%$ p.v. do traçador) e aplicadas, com três diferentes tamanhos de gotas (muito grossa, fina e muito fina) em Hibiscus spp. Após a completa secagem da calda, retiraram-se amostras de folhas, as quais foram levadas ao laboratório e analisadas sob luz ultravioleta; selecionouse, visualmente, uma folha com deposição leve, média e pesada de cada um dos nove tratamentos. A menor dose do traçador proporcionou uma fluorescência bastante incipiente mesmo para análise visual. As demais folhas foram fotografadas com câmera digital, em ambiente totalmente escuro, sob iluminação ultravioleta. Assim, desenvolveu-se uma rotina de análise de imagens que permitiu a automatização das análises de cobertura. $\mathrm{O}$ software mostrou-se ferramenta adequada e eficiente na avaliação da cobertura de pulverização na superfície vegetal estudada. A maior concentração do traçador melhorou a sensibilidade da análise.
\end{abstract}

Palavras-chave: pulverização, distribuição, análise de imagens, traçador fluorescente.

\section{ABSTRACT \\ USING IMAGERY ANALYSIS SOFTWARE FOR EVALUATION OF SPRAYING COVERAGE}

The present work aimed to evaluate the smallest concentration of the tracer fluorescent Poliglow 830 YLS necessary for an adequate contrast with the plant surface, as well as to develop a process for determination of spraying coverage using the software IDRISI for imagery analysis. Three doses of the fluorescent tracer Poliglow 830 YLS (5, 12.5 and $25 \mathrm{~g})$ were previously mixed to $10 \mathrm{~g}$ of a mancozeb formulation, diluted in $5 \mathrm{~L}$ of water $(0.001,0.0025$ e $0.005 \% \mathrm{~W} . \mathrm{v}$. of tracer) and applied on Hibiscus spp, with three different sizes of drops (very large, fine and very fine). After the complete drying of the carrier, samples of leaves were collected, taken to the laboratory and analyzed under ultra-violet light, where a leaf was visually selected with light, medium and heavy deposition, for each one of the nine treatments. The smallest dose of the tracer provided a quite incipient fluorescency for visual analysis, therefore being discarded. The other leaves were photographed with digital camera, in a totally dark atmosphere, under ultra-violet illumination. With these images, a routine analysis was developed in the software IDRISI, which allowed the automation of the coverage analyses. The software IDRISI showed to be an appropriate and efficient tool in the evaluation of the spraying coverage in the plant surface studied. The largest concentration of the tracer improved the sensitivity of the analysis.

Key words: spraying, distribution, imagery analysis, fluorescent tracer.

( $\left.{ }^{1}\right)$ Recebido para publicação em 25 de outubro de 2001 e aceito em 29 de agosto de 2002.

$\left(^{2}\right)$ Graduando em Agronomia. Estagiário do Centro Avançado de Pesquisa Tecnológica do Agronegócio de Engenharia e Automação Agrícola (CMAA), Instituto Agronômico (IAC), Caixa Postal 26, 13201-970 Jundiaí (SP).

$\left(^{3}\right)$ Centro de Mecanização e Automação Agrícola, IAC. E-mail: hhramos@dea.iac.br 


\section{Introdução}

A mistura de aditivos, pó ou líquido, às formulações a serem pulverizadas, como meio de avaliação qualitativa e quantitativa dos depósitos, é bastante antiga. Inicialmente, utilizavam-se os compostos cúpricos; a partir daí, vários outros métodos começaram a ser estudados. Na busca por novos métodos, HIMEL (1969), SHARP (1973), Tompkins et al. citados por Авг SAAB (1996) e EvANs et al. (1994) adicionaram suspensões de pigmentos fluorescentes ao produto a ser aplicado nas plantas, substâncias que se destacam facilmente sob luz ultravioleta pela fluorescência. Segundo RAETANO (1996), a utilização desses materiais fluorescentes apresenta vantagem, pois, sendo invisíveis nas folhas, diminui a tendência de escolha na coleta das amostras; além disso, os depósitos são facilmente visualizados em presença de luz ultravioleta (BLB 15).

Apesar da vantagem do uso dos pigmentos fluorescentes, pode-se citar como desvantagem o fato de, em sua grande maioria, serem pouco solúveis em água. Com isso, faz-se necessário misturá-los previamente aos espalhantes adesivos a fim de melhorar sua dispersão no meio aquoso, como sugerido por RAETANO (1996). Tal mistura, porém, pode afetar os resultados em virtude da redução na tensão superficial da água proporcionada pelo espalhante (PALLADINI, 2000). Considerando esse fato, АBI SAAB (1996) utilizou com sucesso a técnica de misturar o pigmento Luxcor LRM 100 (derivado de uréia/formaldeído) a uma formulação de pó molhável. Segundo esse autor, tal artifício permitiu uma dispersão adequada do pigmento na água, sem a utilização de espalhantes.

Normalmente, o critério adotado para a avaliação dos depósitos, quando se empregam traçadores fluorescentes, tem sido uma escala de notas visual, variável em função da porcentagem ou intensidade de cobertura, conforme descrita por BULLOCK et al. (1968), Palladini (1990) e Raetano (1996). A automação de tais análises, por meio da análise de imagens dos alvos pulverizados por equipamentos eletrônicos, tem sido estudada e foi utilizada com sucesso por Steden citado por ABI SAAB (1996) e EvANS et al. (1994). Essa técnica, entretanto, apresenta limitações, como a necessidade de um grande contraste da imagem para que a avaliação seja precisa (SALYANI et al., 1987). Torna-se importante, contudo, o estudo da concentração do pigmento fluorescente e da rotina de análise para que os resultados obtidos sejam precisos e representem, adequadamente, a cobertura obtida em pulverização.

O presente trabalho teve como objetivo avaliar a menor concentração do traçador fluorescente
Poliglow 830 YLS necessária para um suficiente contraste com a superfície vegetal, bem como desenvolver uma rotina para determinar a cobertura de pulverização no software para análise de imagens IDRISI.

\section{Material e Métodos}

Os experimentos foram realizados no Centro de Mecanização e Automação Agrícola (CMAA), do Instituto Agronômico (IAC), localizado em Jundiaí (SP).

Utilizou-se o pigmento fluorescente Poliglow 830 YLS que, segundo estudos realizados por RAETANO (1996), com diferentes tipos de traçadores, mostrouse mais adequado à avaliação qualitativa da pulverização. Por ser pouco solúvel em água, misturou-se previamente o traçador a uma formulação de mancozeb para dispersão do pigmento - técnica proposta por Аві SАAB (1996). Dessa forma, três doses do pigmento (5, 12,5 e $25 \mathrm{~g})$ foram misturadas a $10 \mathrm{~g}$ da formulação de mancozeb, pesadas em balança digital com precisão de $0,1 \mathrm{~g}$ e acondicionadas em balões volumétricos para transporte até a área experimental.

Para se obter análise mais completa, optou-se por trabalhar com três espectros diferentes de gotas. Para isso, selecionaram-se as pontas de pulverização AI11002, XR11002 e TX-8, da Spraying Systems Co.que, segundo as especificações do fabricante, produzem, respectivamente, gotas muito grossa, fina e muito fina, a 4 bar de pressão (SPRAYING Systems Co., sd).

Os tratamentos constituíram-se da combinação de três concentrações do traçador com três diferentes espectros de gotas, em nove condições.

Realizaram-se as aplicações com um pulverizador costal motorizado marca Guarany, trabalhando à mínima aceleração (pressão de $400 \mathrm{kPa}$ ), sobre plantas de Hibiscus spp. dispostas em 'cerca viva', com $1,5 \mathrm{~m}$ de altura e $50 \mathrm{~m}$ de comprimento. A calda foi preparada em jarra graduada com capacidade de $1 \mathrm{~L}$, onde o volume de água era medido, colocado aos poucos nos balões volumétricos e, posteriormente, no pulverizador até completar $5 \mathrm{~L}$ (concentrações de 0,001, 0,0025 e 0,005\% p.v. do traçador). Para minimizar possíveis interferências, realizou-se uma tríplice lavagem do tanque do pulverizador quando as caldas foram substituídas.

Após 30 minutos da aplicação, tempo suficiente para a secagem da calda, coletaram-se nove folhas de cada parcela (três na parte superior, três na central e três na inferior da planta), totalizando 81 folhas, que foram acondicionadas em sacos plásticos devidamente identificados, e enviadas ao laboratório para análise. 
Em laboratório fotográfico (sem qualquer incidência de luz externa), as folhas coletadas foram analisadas visualmente sob luz ultravioleta; selecionaram-se para a análise, três folhas de cada parcela, com diferentes graus de cobertura (pesada, média e leve). A menor dose, $5 \mathrm{~g}$ de Poliglow 830 YLS (0,001\% p.v.), foi insuficiente para possibilitar um contraste adequado entre a deposição e a folha para análise visual, sendo descartada na análise eletrônica. Doses de $12,5 \mathrm{~g}(0,0025 \%$ p.v.) ou superior mostraram-se adequadas a essa análise.

Para digitalização das imagens, as folhas selecionadas foram postas sobre um suporte de vidro, sob o qual se colocou um papel branco para aumentar o contraste do fundo com a imagem. Duas lâmpadas ultravioletas (BLB-FL-15W-T8) foram posicionadas a $15 \mathrm{~cm}$ de altura em relação à posição das folhas e $20 \mathrm{~cm}$ de distância entre si. As imagens foram captadas por câmera fotográfica digital Kodak modelo DC120, posicionada perpendicularmente a uma distância aproximada de $70 \mathrm{~cm}$ da folha (mínima distância focal possível), com o máximo de zoom (3x), velocidade de abertura 1:1 e com o flash desligado. As imagens obtidas foram transferidas para um computador no formato JPG e, posteriormente, transformadas em BMP para possibilitar a análise no software IDRISI.

De acordo com EASTMAN Jr (1997), o IDRISI é um sistema de informação geográfica e um software para processamento de imagens; líder na funcionalidade analítica raster, cobrindo todo o espectro de necessidade de sistema de informação geográfica (SIG) e de sensoriamento remoto, desde a consulta ao banco de dados e modelagem espacial até realce e classificação de imagens, possibilitando, também,

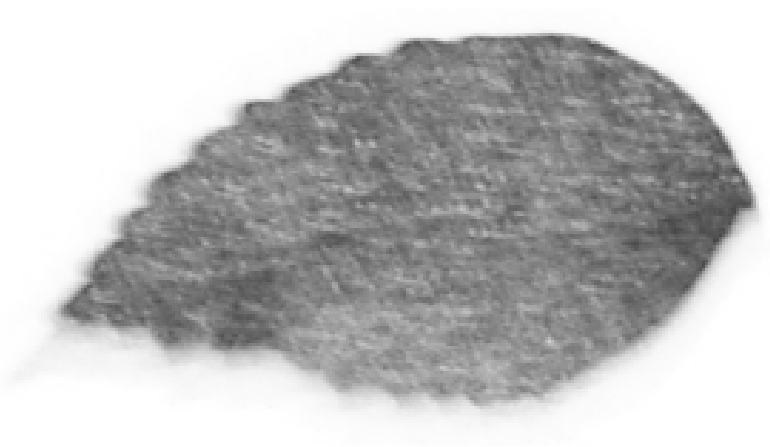

(A) pela divergência de cores, calcular diferenças de áreas em uma mesma superfície. As características de realce e classificação da imagem, bem como o cálculo de áreas foram utilizados no desenvolvimento da rotina para avaliação da cobertura de pulverização da superfície foliar.

\section{Resultados e Discussão}

Para a análise das imagens no IDRISI, dentre as diferentes opções disponíveis nas tabelas de cores, o modo 'Color Composit 256', que divide o espectro em um conjunto de 256 cores, proporcionou maior precisão nos limites entre folha, cobertura e fundo (Figura 1). Observou-se que, nesse modo de exibição, a imagem foi composta por um conjunto de, aproximadamente, 25 cores, e os conjuntos que formavam a folha, a cobertura e o fundo foram distintos. Utilizando o 'Reclass', que permite reclassificar como única cor toda uma faixa de cores, foi possível agrupar os diferentes conjuntos em apenas três cores. Assim, as cores de 0 a 55 foram agrupadas no Grupo I; as de 56 a 178 no Grupo II; as de 179 a 256 no Grupo III, correspondentes, respectivamente, à folha, deposição e fundo (Figura 1). Nas deposições leves ou muito baixas, a faixa de folha por vezes se confundia com a cobertura. Nesse caso, as cores foram inicialmente reunidas em cinco diferentes grupos e, posteriormente, utilizou-se novamente o 'Reclass' para se obter apenas os três pretendidos. Obtida a imagem, utilizou-se o analisador de áreas para conseguir, em pixels, a área correspondente a cada uma das cores. A área coberta foi calculada em vista da porcentagem da área da calda em relação à área da folha + calda.

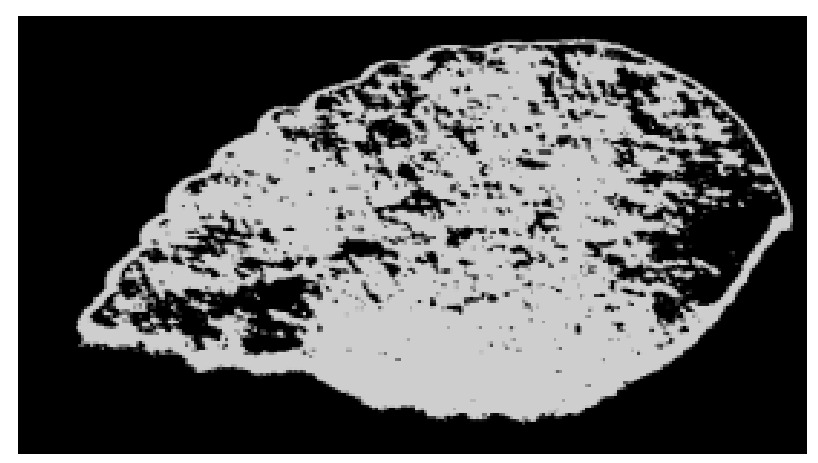

(B)

Figura 1. Imagem de folha pulverizada com traçador fluorescente e analisada no software IDRISI sob a paleta de cores 'Color Composit 256' (A) e, após, reclassificada em fundo (cinza-escuro), folha (preto) e cobertura (cinza-claro), visualizada sob a paleta de cores 'Qualitative 16' (B). 
Quadro 1. Resultados das análises de cobertura da folha pela calda de pulverização, realizadas de forma visual e pela rotina desenvolvida para esta finalidade com o uso do software IDRISI, para a concentração de $0,0025 \%$ p.v. do traçador

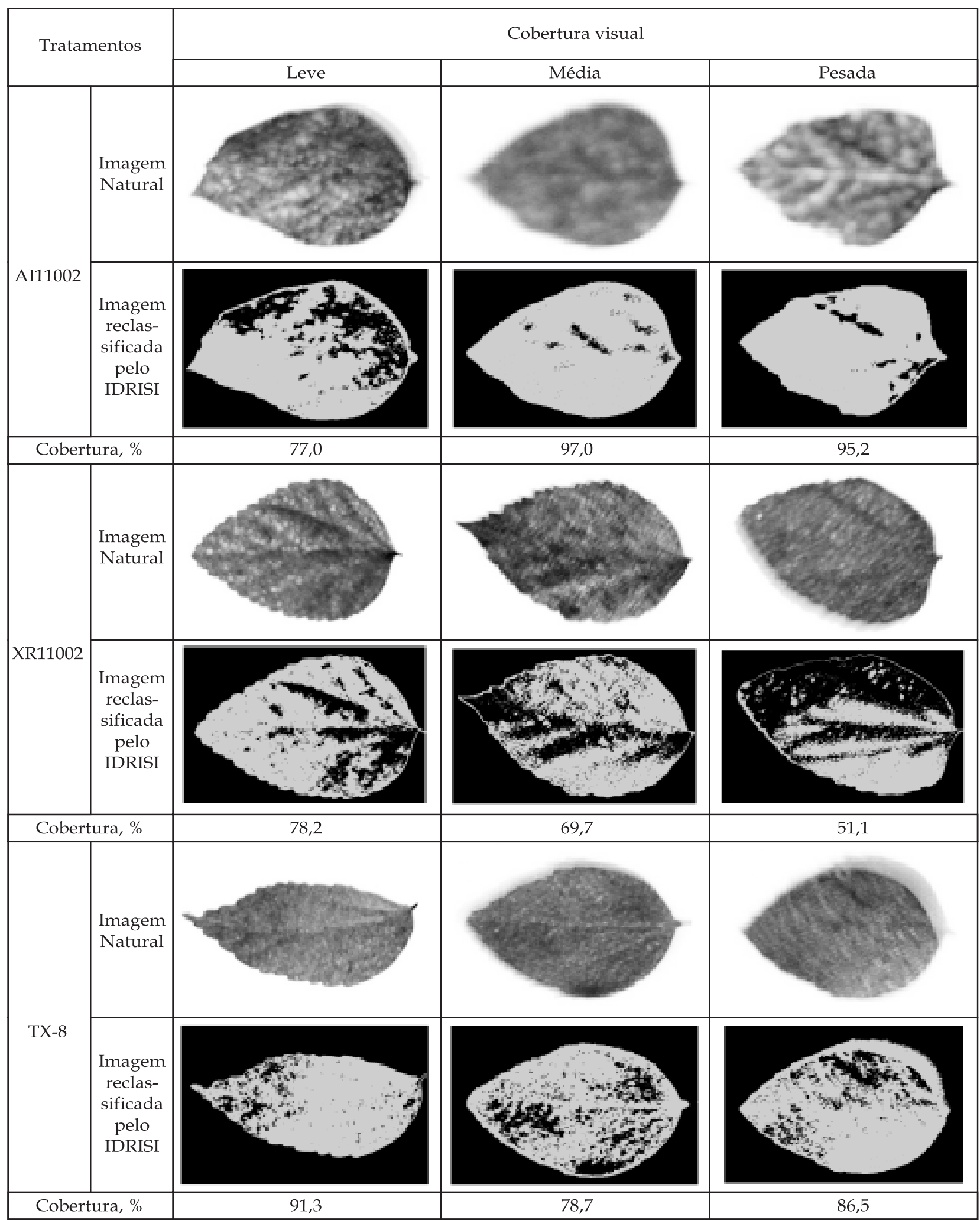

Escala de cores: fundo (cinza-escuro), folha (preto) e cobertura (cinza-claro) 
Quadro 2. Resultados das análises de cobertura da folha pela calda de pulverização, realizadas de forma visual e pela rotina desenvolvida para esta finalidade com o uso do software IDRISI, para a concentração de 0,005\% p.v. do traçador

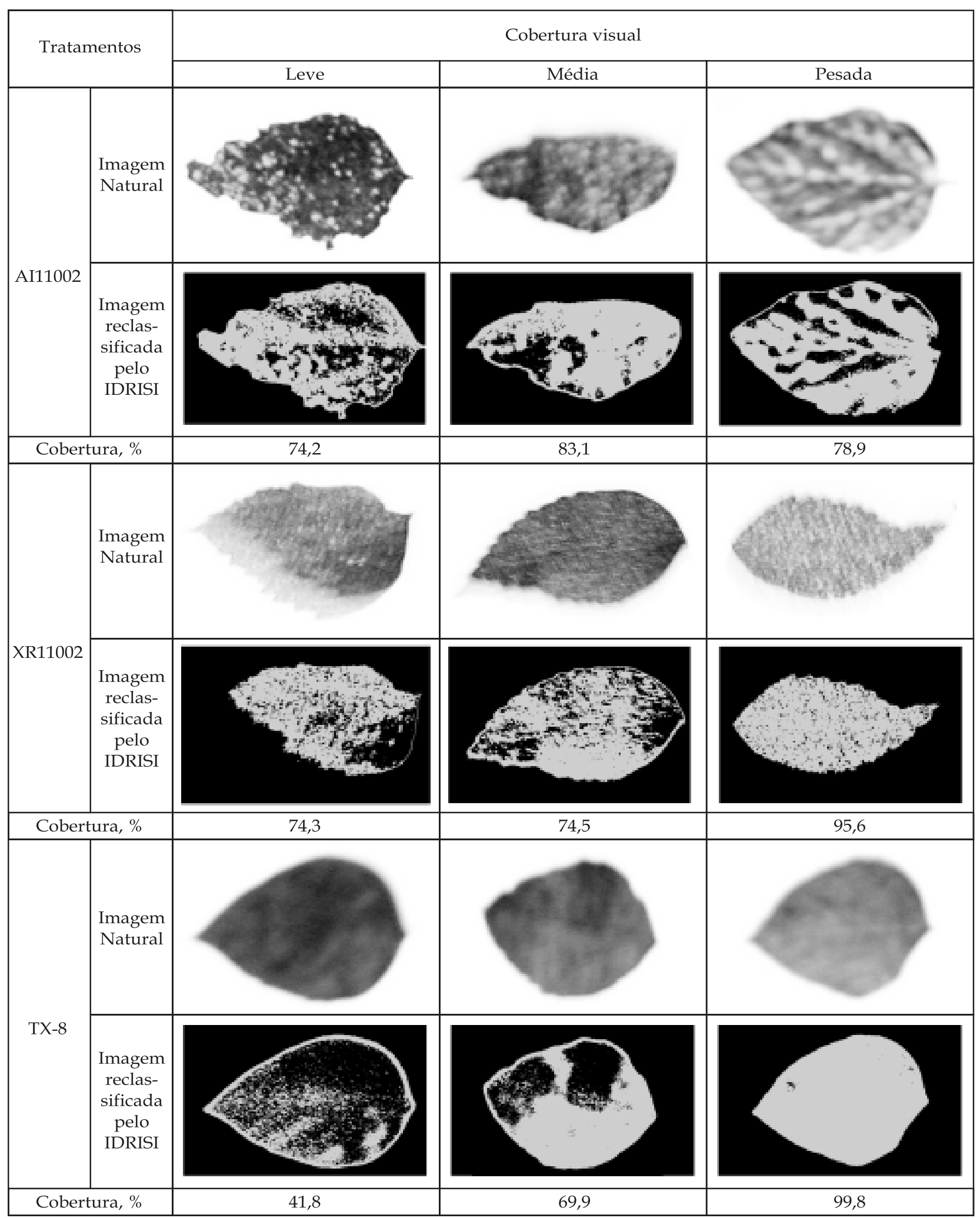

Escala de cores: fundo (cinza-escuro), folha (preto) e cobertura (cinza-claro) 
A rotina de análise desenvolvida no IDRISI mostrou-se eficiente no cálculo da área coberta, apresentando resultados diferentes ao obtidos de forma visual (Quadros 1 e 2). Para as coberturas consideradas visualmente leves, médias e pesadas, as análises variaram entre $77 \%$ e $91 \%$; $69 \%$ e $97 \%$; $51 \%$ e $95 \%$, respectivamente, para a menor concentração do traçador $(0,0025 \%$ p.v. $)$, quando avaliadas pela rotina desenvolvida no IDRISI. Para maior concentração do traçador $(0,005 \%$ p.v. $)$, as coberturas determinadas pelo IDRISI variaram, respectivamente, entre $41 \%$ e $74 \% ; 79 \%$ e $83 \%$; $79 \%$ e $100 \%$. Observa-se, na menor concentração do traçador, que a diferença entre a análise visual e a automatizada foi elevada. Atribuem-se tais diferenças, principalmente às áreas de baixa cobertura, com conseqüente baixa luminescência, o que dificulta a visualização. Na maior concentração, além da diferença entre as formas de análise ser menor, a amplitude de variação dentro de uma mesma classe também foi reduzida. Tal fato se deve ao maior contraste entre a área coberta e a folha, em concordância com o observado por SALYANI et al. (1987). Portanto, o aumento na concentração do traçador, ou mesmo a utilização de um outro que emita fluorescência em uma faixa bem distinta do verde, pode simplificar e elevar a eficiência da operação.

Outro fator limitante observado foi a não-fixação da câmera durante a aquisição das fotos. Quando isso ocorreu, devido ao longo tempo de abertura do diafragma, formou-se um halo, mais perceptível nas bordas das folhas, o qual, algumas vezes, dificultou a identificação da área de transição. Resolveu-se tal problema com a fixação da câmera sobre um tripé apropriado.

\section{Conclusões}

1. O Poliglow 830 YLS, quando misturado ao mancozeb em formulação pó molhável e doses superiores a 12,5 g por 5 litros de água $(0,0025 \%$ p.v.) na calda de pulverização, possibilita um contraste suficiente para a análise digital dos resultados.

2. A elevação na quantidade do traçador, de 12,5 para 25,0 g por 5 litros $(0,0025 \%$ p.v. e $0,005 \%$ p.v. respectivamente), diminuiu a diferença entre o sistema visual e o automatizado de análise, além de reduzir a diferença entre a maior e a menor cobertura dentro de uma mesma classe (leve, média ou pesada).

3. O software IDRISI mostrou-se ferramenta adequada e eficiente na avaliação da cobertura proporcionada pela pulverização na superfície vegetal estudada.

\section{REFERÊNCIAS BIBLIOGRÁFICAS}

ABI SAAB, O.J.G. Avaliação de um sistema de aplicação de defensivos utilizado em videiras no município de Londrina/PR. 1996. 65f. Dissertação (Mestrado em Agronomia) - Faculdade de Ciências Agronômicas - UNESP, Botucatu.

BULLOCK, R.C.; BROOKS, R.F.; WHITNEY, J.D. A method of evaluating pesticide application equipment for Florida citrus. Journal of Economic Entomology, Lanham, v.61, n.6, p.1511-1514, 1968.

EASTMAN Jr. Idrisi for windows: user's guide, version 2.0. Clark University, 1997. 350p.

EVANS, M.D.; LAW, S.E.; COOPER, S.C. Fluorescent spray deposit measurement via light intensified machine vision. Applied Engineering in Agriculture, St. Joseph, v.10, n.4, p.463-470, 1994.

HIMEL, C.M. The fluorescent particle spray droplet tracer method. Journal of Economic Entomology, Lanham, v.62, n.4, p.912-916, 1969.

PALLADINI, L.A. Efeito de condições operacionais de um turboatomizador na cobertura de folhas de citros. 1990. 93f. Dissertação (Mestrado em Agronomia) Faculdade de Ciências Agrárias e Veterinárias, UNESP, Jaboticabal.

PALLADINI, L.A. Metodologia para avaliação da deposição em pulverizações. 2000. 111f. Tese (Doutorado em Agronomia) - Faculdade de Ciências Agronômicas, UNESP, Botucatu.

RAETANO, C.G. Condições operacionais de turboatomizadores na distribuição e deposição da pulverização em citros. 1996. 93f. Tese (Doutorado em Agronomia). Escola Superior de Agricultura "Luiz de Queiroz" - USP, Piracicaba.

SALYANI, M.; HEDDEN, S.L.; EDWARDS, G.J. Deposition efficiency of different droplet sizes for citrus spraying. Transactions of the Asae, St. Joseph, v.30, n.6, p.1595-1599, 1987.

SHARP, R.B. A rapid method of spray deposit measurement and its use in new apple orchards. In: BRITISH INSECTICIDE AND FUNGICIDE CONFERENCE, 7., Brighton, 1973. Proceedings... Brighton, 1973. p.637-641.

SPRAYING SYSTEMS CO. Produtos de pulverização para agricultura. São Paulo, s.d., 104p. (CAT. 46M-BR/P). 